\title{
Groups of Automorphisms with Spectrum Condition and the Lifting Problem
}

\author{
Dorte Olesen and Gert Kjærgård Pedersen \\ Institute of Mathematics, University of Copenhagen, DK-2100 Copenhagen, Denmark
}

\begin{abstract}
We show that a continuous one-parameter group $\left\{\alpha_{t}\right\}$ of automorphisms of a separable $C^{*}$-algebra $A$ satisfies a spectrum condition if and only if it is the limit, pointwise on $A$, uniformly on compact subsets of $\mathbb{R}$ of a sequence of inner automorphism groups whose positive generators do not increase too fast. Moreover we prove in this case that if $\pi$ is a surjective morphism of a separable $C^{*}$-algebra $B$ on $A$ then there is a similar group $\left\{\bar{\alpha}_{t}\right\}$ of automorphisms of $B$ such that $\pi \circ \bar{\alpha}_{t}=\alpha_{t} \circ \pi$ for all $t$.
\end{abstract}

\section{Introduction}

Most of the current work in operator algebra is influenced by problems arising in mathematical physics and is concerned with situations involving a $C^{*}$-algebra $A$ (the observables) and a continuous one-parameter group $\left\{\alpha_{t}\right\}$ of automorphisms of $A$ (the time development). We can always represent $A$ as operators on some Hilbert space $H$ such that $\alpha_{t}(x)=u_{t} x u_{t}^{*}$ for all $x$ in $A$ and $t$ in $\mathbb{R}$, where $u_{t}=\exp (i t h)$ and $h$ is a self-adjoint operator (the hamiltonian) in $H$. The case where $h$ is (or can be chosen to be) semi-bounded is interesting for the applications (corresponding to positive energy) and quite attractive from a mathematical point of view. We say in this situation that $\left\{\alpha_{t}\right\}$ satisfies a spectrum condition in the given representation of $A$.

In this paper we deal with the most restrictive version of the above, namely that $\left\{\alpha_{t}\right\}$ satisfies a spectrum condition in the universal representation of $A$. Unfortunately, this assumption is not tenable in more realistic models, so our results must be taken more as a guideline what to expect, than as a valid description of a physical situation. We show that there is an increasing (but unbounded) sequence $\left\{h_{n}\right\}$ in $A_{+}$which converges (in a sense to be made precise) such that the sequence $\left\{\exp \left(i t h_{n}\right) \cdot \exp \left(-i t h_{n}\right)\right\}$ of inner automorphism groups converges to $\left\{\alpha_{t}\right\}$ pointwise on $A$ and uniformly on compact subsets of $\mathbb{R}$.

The paper originated in an effort to extend the lifting theorem for automorphism groups to groups satisfying a spectrum condition. It was shown in 
[9, Cor. 2] (see also [10, Th. 1]) that if $\left\{\alpha_{t}\right\}$ is uniformly continuous on $A$ and $\pi$ is a surjective morphism from a separable $C^{*}$-algebra $B$ onto $A$ then there is a uniformly continuous group $\left\{\bar{\alpha}_{t}\right\}$ on $B$ such that $\pi \circ \bar{\alpha}_{t}=\alpha_{t} \circ \pi$ for all $t$ in $\mathbb{R}$. The proof of this result relies heavily on the theory of spectral subspaces as developed by Arveson and Borchers (see e.g. [2-4] and [6]); and since that theory works equally well for a continuous group $\left\{\alpha_{t}\right\}$ provided that it satisfies a spectrum condition, it was natural to conjecture that the lifting problem could be solved also in this situation.

We prove in this paper that such is indeed the case: A one-parameter group with spectrum condition can be lifted. To appreciate the result one may consult the rather disappointing list of counter examples in [10]. As in [9] and [10] the proof consists of lifting a derivation from a quotient. However, the derivation is now unbounded so that the lifted one is not a priori the infinitesimal generator for a group, and this additional difficulty makes the argument considerably more involved.

The authors wish to record their gratitude to D. Kastler for his hospitality at Universite de Marseille, U.E.R. Luminy, where part of this work was completed, and to O. Bratteli, D.W. Robinson and H. Araki for stimulating conversations.

\section{Preliminaries}

A one-parameter group $\left\{\alpha_{t} \mid t \in \mathbb{R}\right\}$ of automorphisms (i. e. *-automorphisms) of a $C^{*}$-algebra $A$ is continuous if the function $t \rightarrow \alpha_{t}(x)$ is continuous from $\mathbb{R}$ to $A$ for each $x$ in $A$. There is then a densely defined skew-adjoint derivation $\delta$ of $A$ such that $\alpha_{t}=\exp (i t \delta)$. We say that an element $x$ in $A$ is smooth (relative to $\left\{\alpha_{t}\right\}$ ) if $x$ belongs to the domain of $\delta^{n}$ for all $n$. If $f$ is a $C^{\infty}$-function on $\mathbb{R}$ with compact support then for each $x$ in $A$ the element $y=\int \alpha_{t}(x) f(t) d t$ is smooth since $i \delta(y)=$ $\int \alpha_{t}(x) f^{\prime}(t) d t$. Thus the set of smooth elements is a dense *-subalgebra of $A$.

If $A$ is represented as operators on some Hilbert space $H$ we say that the group $\left\{\alpha_{t}\right\}$ has a unitary implementation on $H$ if there is a self-adjoint operator $h$ (possibly unbounded) such that $\alpha_{t}(x)=\exp (i t h) x \exp (-i t h)$ for all $x$ in $A$ and $t$ in $\mathbb{R}$. In this case we have the following characterization of the infinitesimal generator $\delta$ of $\left\{\alpha_{t}\right\}$ :

Lemma A. If $x \in A$ then $x \in \mathscr{D}(\delta)$ if and only if

(i) $x \mathscr{D}(h) \subset \mathscr{D}(h)$,

(ii) The operator $h x-x h$ on $\mathscr{D}(h)$ has an extension (necessarily unique) to an element in $A$ denoted by $[h, x]$.

Moreover, if $x \in \mathscr{D}(\delta)$ then $\delta(x)=[h, x]$.

Proof. Take $x$ in $\mathscr{D}(\delta)$, and $\xi$ in $\mathscr{D}(h)$. Then since

$$
\begin{aligned}
(i t)^{-1}\left(\alpha_{t}(x)-x\right) \xi & =(i t)^{-1}(\exp (i t h) x \exp (-i t h)-x) \xi \\
& =\exp (i t h)(i t)^{-1}[x(\exp (-i t h)-1) \xi-(\exp (-i t h)-1) x \xi],
\end{aligned}
$$

we obtain in the limit

$$
\delta(x) \xi=-x h \xi-\lim (i t)^{-1}(\exp (-i t h)-1) x \xi .
$$

This shows that $x \xi \in \mathscr{D}(h)$ and moreover that $\delta(x)$ is an extension of $h x-x h$ from $\mathscr{D}(h)$. 
To prove the converse define for each $a$ in $A$

$$
I_{s}(a)=\int_{0}^{s} \alpha_{t}(a) d t \text {. }
$$

Note that $I_{s}(a) \in \mathscr{D}(\delta)$ with $\delta\left(I_{s}(a)\right)=i^{-1}\left(\alpha_{s}(a)-a\right)$. Thus from the first half of the proof we obtain

$$
i^{-1}\left(\alpha_{s}(x)-x\right) \xi=\left(h I_{s}(x)-I_{s}(x) h\right) \xi
$$

for each $\xi$ in $\mathscr{D}(h)$. Assuming that $x$ satisfies (i) we have

$$
i^{-1}\left(\alpha_{s}(x)-x\right) \xi=\int_{0}^{s} \exp (i t h)(h x-x h) \exp (-i t h) \xi d t
$$

because $h$ commutes with $\exp (i h t)$. If furthermore $x$ satisfies (ii) then with $[h, x]$ as the extension of $h x-x h$ we get

$$
i^{-1}\left(\alpha_{s}(x)-x\right)=I_{s}([h, x]) \text {. }
$$

Since $\left\{\alpha_{t}\right\}$ acts continuously on $A$ we have $\lim s^{-1} I_{s}(a)=a$ (in norm) for each $a$ in $A$; in particular

$$
\lim (i s)^{-1}\left(\alpha_{s}(x)-x\right)=[h, x],
$$

whence $x \in \mathscr{D}(\delta)$ with $\delta(x)=[h, x]$.

Regarding $A$ as an algebra of operators on its universal Hilbert space $H$ (see $[5, \S 12]$ ) we say that the group $\left\{\alpha_{t}\right\}$ satisfies a universal spectrum condition if it can be implemented by a unitary group $\{\exp (i t h)\}$ where $h$ is a self-adjoint operator whose spectrum is contained in a halfline (which we take as $\mathbb{R}_{+}$without loss of generality). A "space-free" characterization of the spectrum condition was obtained in $[4, \S 3]$ : For a norm dense set of states $\omega$ of $A$ each function $t \rightarrow \omega\left(x \alpha_{t}(y)\right)$ has an analytic extension of exponential type in the upper half plane. The theory of spectral subspaces shows that when $\left\{\alpha_{t}\right)$ satisfies a spectrum condition then there is a canonical implementing group $\left\{u_{t}\right\}$ in the enveloping von Neumann algebra $A^{\prime \prime}$ of $A$. Moreover, as seen from [6, Prop. 3.5.7] the infinitesimal generator $h$ of $\left\{u_{t}\right\}$ (which is a positive operator affiliated with $A^{\prime \prime}$ ) is the minimal positive generator for any positive implementing group $\left\{v_{t}\right\}$. This means that if

$$
u_{t}=\int_{0}^{\infty} \exp (i t \lambda) d p(\lambda) \text { and } v_{t}=\int_{0}^{\infty} \exp (i t \lambda) d q(\lambda)
$$

then $p([0, \lambda]) \geqq q([0, \lambda])$ for every $\lambda$ (or, formally, that $h^{n} \leqq k^{n}$ for all $n$ ). For the von Neumann algebra version of this see [2, p. 235].

It will be convenient, as in $[11, \S 4]$, to extend the notions of order and monotone strong convergence to unbounded positive operators. For each $\varepsilon$ in $\mathbb{R}$ define $f_{\varepsilon}$ on $\left[0, \infty\right.$ [ if $\varepsilon \geqq 0$ and on $\left[0,-\varepsilon^{-1}\left[\right.\right.$ if $\varepsilon<0$ by $f_{\varepsilon}(t)=t(1+\varepsilon t)^{-1}$. Each of these functions is operator monotone on their interval of definition [i.e. if $x$ and $y$ are positive bounded operators and $x \leqq y$ then $f_{\varepsilon}(x) \leqq f_{\varepsilon}(y)$, provided that $\|y\|<-\varepsilon^{-1}$ if $\left.\varepsilon<0\right]$. Moreover, $f$ is bounded (by $\varepsilon^{-1}$ ) when $\varepsilon>0$, and $f_{\delta}^{\circ} f_{\varepsilon}=f_{\delta+\varepsilon}$ if $\varepsilon \geqq 0$ and $\delta+\varepsilon \geqq 0$. In particular, $f_{-\varepsilon} \circ f_{\varepsilon}$ is the identity map on $\left[0, \infty\left[\right.\right.$ so that $x \leqq y$ if and only if $f_{\varepsilon}(x) \leqq f_{\varepsilon}(y)$.

If $x$ and $y$ are self-adjoint, positive operators (possibly unbounded) on a Hilbert space we define $x \leqq y$ to mean that $f_{\varepsilon}(x) \leqq f_{\varepsilon}(y)$ for some and hence any $\varepsilon>0$. Furthermore, if $\left\{x_{n}\right\}$ is an increasing sequence (in this extended sense) of 
self-adjoint, positive operators we write $x_{n}>x$ if $\left\{f_{\varepsilon}\left(x_{n}\right)\right\}$ is strongly convergent to $f_{\varepsilon}(x)$ for some positive, self-adjoint operator $x$ and some (hence any) $\varepsilon>0$. It is straightforward to verify from these definitions that if $\left\{x_{n}\right\}$ is an increasing sequence of positive, self-adjoint operators such that $x_{n} \leqq y$ for some self-adjoint operator $y$ and all $n$, there is a unique self-adjoint operator $x$ such that $x_{n} \nearrow x$, and $x \leqq y$.

These notions of order and monotone convergence coincide with the ones obtained by representing a self-adjoint, positive operator as a quadratic form (see [11, Lemma 7.9]) but some difficulties concerning domains and closedness are avoided by our approach.

The following lemma can be read out of [1, Lemma 3.3]. For convenience we include a proof.

Lemma B. If $\left\{x_{n}\right\}$ is an increasing sequence of positive, self-adjoint (possibly unbounded) operators such that $x_{n}>x$ for some self-adjoint operator $x$, then $\left\{f\left(x_{n}\right)\right\}$ converges strongly to $f(x)$ for each bounded continuous function $f$ on $\mathbb{R}_{+}$.

Proof. Straightforward calculations show that the set $L$ of functions in $C^{b}\left(\mathbb{R}_{+}\right)$ satisfying the requirements of the lemma is a $C^{*}$-subalgebra of $C^{b}\left(\mathbb{R}_{+}\right)$. Since $L$ contains the constants and all functions $f_{\varepsilon}$ with $\varepsilon>0$ we conclude from the StoneWeierstrass theorem that $L$ contains all of $C\left(\mathbb{R}_{+} \cup\{\infty\}\right)$.

Take now $f$ in $C^{b}\left(\mathbb{R}_{+}\right)$. For each vector $\xi$ and each $\varepsilon>0$ there is a continuous function $g$ on $\mathbb{R}_{+}$with compact support such that $\|g(x) \xi-\xi\|<\varepsilon$. But then

$$
\begin{aligned}
\left\|\left(f(x)-f\left(x_{n}\right)\right) \xi\right\| \leqq & \|(f(x)-f(x) g(x)) \xi\| \\
& +\left\|\left(f(x) g(x)-f\left(x_{n}\right) g\left(x_{n}\right)\right) \xi\right\|+\left\|\left(f\left(x_{n}\right) g\left(x_{n}\right)-f\left(x_{n}\right)\right) \xi\right\| \\
\leqq & \|f\| \varepsilon+\left\|\left(f \cdot g(x)-f \cdot g\left(x_{n}\right)\right) \xi\right\|+\|f\|\left\|g\left(x_{n}\right) \xi-\xi\right\| .
\end{aligned}
$$

Since both $g$ and $f \cdot g$ belong to $L$ we conclude that

$$
\lim \sup \left\|\left(f(x)-f\left(x_{n}\right)\right) \xi\right\|<2\|f\| \varepsilon,
$$

which implies that $\left\{f\left(x_{n}\right)\right\}$ converges strongly to $f(x)$. Hence $f \in L$ and $L=C^{b}\left(\mathbb{R}_{+}\right)$ as desired.

\section{Some Lemmas}

Lemma 1. Let $\left\{\alpha_{t}\right\}$ be a continuous one-parameter group of automorphisms of a separable $C^{*}$-algebra A. If $\left\{\alpha_{t}\right\}$ satisfies a spectrum condition and $h$ is the minimal positive generator for $\left\{\alpha_{t}\right\}$ then

$$
\left[f_{\varepsilon}(h), a\right] \rightarrow[h, a] \quad \text { as } \quad \varepsilon \rightarrow 0
$$

strongly, for every smooth element $a$ in $A$.

Proof. The following formula is easily verified (but not so easily found):

$$
\begin{aligned}
& {[h, a]-\left[f_{\varepsilon}(h), a\right]} \\
& \quad=\varepsilon h(1+\varepsilon h)^{-1}[h, a](1+\varepsilon h)^{-1}+(1+\varepsilon h)^{-1}[h, a] \varepsilon h(1+\varepsilon h)^{-1} \\
& \quad+\varepsilon h(1+\varepsilon h)^{-1}[h, a] \varepsilon h(1+\varepsilon h)^{-1} .
\end{aligned}
$$


Here $[h, a] \in A,(1+\varepsilon h)^{-1} \rightarrow 1$ strongly, $\varepsilon h(1+\varepsilon h)^{-1} \rightarrow 0$ strongly and the Lemma follows.

Lemma 2. Under the same assumptions as in Lemma 1 there is an increasing sequence $\left\{x_{n}\right\}$ in $A_{+}$consisting of smooth elements such that $x_{n}>h(1+h)^{-1}$ and $\left\|\left[h, x_{n}\right]\right\| \rightarrow 0$.

Proof. As shown in the proof of [6, Prop. 3.5.3] each spectral projection of $h$ corresponding to an interval ] $t, \infty$ [ is the support of the spectral subspace $R^{\alpha}(t, \infty)$ and therefore open by [6, Lemma 3.3.4]. Thus each spectral projection of the bounded operator $f_{1}(h)=h(1+h)^{-1}$ corresponding to an interval $] t, \infty$ [ is open. Since $f_{1}(h)$ is the norm limit of convex combinations of such projections (see e.g. the proof of [7,Th. 2.1] we conclude that $f_{1}(h)$ is a lower semi-continuous element in $A^{\prime \prime}$. The separability of $A$ then implies that there is an increasing sequence $\left\{y_{n}\right\}$ in $A_{+}$converging strongly to $f_{1}(h)$. Choose a positive $C^{\infty}$-function $f$ on $\mathbb{R}$ with compact support and integral one and define

$$
z_{n}=\int \alpha_{t}\left(y_{n}\right) f(t) d t \in A_{+} .
$$

Each $z_{n}$ is smooth and since $\alpha_{t}\left(f_{1}(h)\right)=f_{1}(h)$ for all $t$ we conclude that $z_{n} \nearrow f_{1}(h)$. Moreover,

$$
\left[h, z_{n}\right]=\int \alpha_{t}\left(y_{n}\right) f^{\prime}(t) d t \rightarrow \int f_{1}(h) f^{\prime}(t) d t=0
$$

(strong convergence). In particular the sequence $\left\{\left[h, z_{n}\right]\right\}$ is $\sigma$-weakly convergent to zero. But the $\sigma$-weak topology of $A$ in its universal representation is the $\sigma\left(A, A^{\prime}\right)$ topology and therefore has the same closed convex sets as the norm topology. Consequently the convex hull of $\left\{\left[h, z_{n}\right]\right\}$ contains zero as a limit point in norm. We can therefore find a sequence $\left\{x_{n}\right\}$ in $A_{+}$such that $x_{n}=\sum \alpha_{i} z_{i}, \alpha_{i} \geqq 0$ and $\sum \alpha_{i}=1$ for each $n$, and such that

$$
\left\|\left[h, x_{n}\right]\right\|=\left\|\sum \alpha_{i}\left[h, z_{i}\right]\right\|<n^{-1} .
$$

Taking each $x_{n}$ as a convex combination of elements $z_{i}$ such that $i$ is greater than any index used in the definition of the previous $x_{k}, k<n$, we obtain an increasing sequence of smooth elements such that $x_{n}>f_{1}(h)$ and $\left\|\left[h, x_{n}\right]\right\|<n^{-1}$ for all $n$.

Lemma 3. There is a sequence $\left\{x_{n}\right\}$ satisfying the requirements in Lemma 2 such that for each $n$ and each $\varepsilon>0$ the element $f_{\varepsilon-1}\left(x_{n}\right)$ is smooth and $\left\|\left[h, f_{\varepsilon-1}\left(x_{n}\right)\right]\right\| \leqq 2^{-n}$.

Proof. Choose the sequence $\left\{x_{n}\right\}$ from Lemma 2 such that $\left\|\left[h, x_{n}\right]\right\| \leqq n^{-2} 2^{-n}$ for all $n$. Multiplying if necessary each element with $1-n^{-1}$ (a process which preserves all properties of $\left.\left\{x_{n}\right\}\right)$ we may assume that $\left\|x_{n}\right\| \leqq 1-n^{-1}$ for all $n$. Then

$$
f_{\varepsilon-1}\left(x_{n}\right)=x_{n}\left(1-(1-\varepsilon) x_{n}\right)^{-1}=\sum_{k=1}^{\infty}(1-\varepsilon)^{k-1} x_{n}^{k} .
$$

Moreover, since $\operatorname{ad}(h)$ is a closed operator

$$
\left[h, f_{\varepsilon-1}\left(x_{n}\right)\right]=\sum_{k=1}^{\infty} \sum_{p=1}^{k}(1-\varepsilon)^{k-1} x_{n}^{p-1}\left[h, x_{n}\right] x_{n}^{k-p} .
$$

This series, as well as any of series obtained by subsequent derivation with $h$, is convergent. It follows that $f_{\varepsilon-1}\left(x_{n}\right)$ is smooth and by summing the series above we get

$$
\left\|\left[h, f_{\varepsilon-1}\left(x_{n}\right)\right]\right\| \leqq \sum_{k=1}^{\infty} k\left\|x_{n}\right\|^{k-1}\left\|\left[h, x_{n}\right]\right\|=\left(1-\left\|x_{n}\right\|\right)^{-2}\left\|\left[h, x_{n}\right]\right\| \leqq 2^{-n} .
$$


Lemma 4. Under the same assumptions as in Lemma 1 and given a sequence $\left\{a_{k}\right\}$ of smooth elements in $A$, there is an increasing sequence $\left\{h_{n}\right\}$ in $A_{+}$consisting of smooth elements such that $h_{n}$ Th, and for all $k \leqq n$

$$
\left\|\left[h-h_{n}, a_{k}\right]\right\| \leqq 2^{-n} \text { and }\left\|\left[h-h_{n}, h_{k}\right]\right\| \leqq 2^{-n} .
$$

Proof. For each finite set $\left\{a_{k}\right\}$ of smooth elements in $A$, each functional $\omega$ in $A^{\prime}$ and each $\delta>0$ there is by Lemma 1 an $\varepsilon>0$ such that

$$
\left|\omega\left(\left[h, a_{k}\right]-\left[f_{\varepsilon}(h), a_{k}\right]\right)\right|<\delta
$$

for all $k$. But for fixed $\varepsilon>0$ and $\left\{x_{n}\right\}$ as in Lemma 3 we have

$$
f_{\varepsilon-1}\left(x_{n}\right) \nearrow f_{\varepsilon-1}\left(f_{1}(h)\right)=f_{\varepsilon}(h)
$$

whence, for sufficiently large $n$,

$$
\left|\omega\left(\left[h, a_{k}\right]-\left[f_{\varepsilon-1}\left(x_{n}\right), a_{k}\right]\right)\right|<\delta
$$

for all $k$.

With $X$ as the direct sum of card $\left\{a_{k}\right\}$ copies of $A$ we conclude from the above that the set of elements in $X$ of the form

$$
\left\{\oplus\left(\left[h, a_{k}\right]-\left[f_{\varepsilon-1}\left(x_{n}\right), a_{k}\right]\right)\right\}
$$

contains zero as a limit point in the $\sigma\left(X, X^{\prime}\right)$-topology. Its convex hull therefore contains zero as a limit point in norm on $X$. We can thus for each $n$ find a smooth element in $A_{+}$of the form

$$
h_{n}=\sum \alpha_{i} f_{\varepsilon_{i}-1}\left(x_{i}\right) \text { with } \sum \alpha_{i}=1
$$

such that

$$
\left\|\left[h-h_{n}, a_{k}\right]\right\|=\left\|\sum \alpha_{i}\left[h-f_{\varepsilon_{l}-1}\left(x_{i}\right), a_{k}\right]\right\| \leqq 2^{-n}
$$

for all $k$.

Working inductively we may assume that

$$
\left\|\left[h-h_{n}, a_{k}\right]\right\| \leqq 2^{-n} \text { and }\left\|\left[h-h_{n}, h_{k}\right]\right\| \leqq 2^{-n}
$$

for all $a_{k}$ in our fixed sequence with $k \leqq n$, and all previous $h_{k}$ with $k<n$. We may further construct the sequence $\left\{h_{n}\right\}$ such that the $\varepsilon$ 's used in the definition of $h_{n}$ all are smaller then $n^{-1}$ and also smaller than those used in any of the previous $h_{k}$; and that the $i$ 's used in the definition of $h_{n}$ all are greater than $n$ and also greater than any of the indices used in the previous $h_{k}$. In this way we make sure that

$$
h_{k}=\sum \alpha_{j} f_{\varepsilon_{j}-1}\left(x_{j}\right) \leqq \sum \alpha_{i} f_{\varepsilon_{i}-1}\left(x_{i}\right)=h_{n}
$$

whenever $k<n$. We also get

$$
x_{n}\left(1-\left(1-n^{-1}\right) x_{n}\right)^{-1} \leqq h_{n} \leqq h
$$

for all $n$, from which we see that $h_{n} \pi h$. Finally, from Lemma 3 we see that

$$
\left\|\left[h, h_{n}\right]\right\| \leqq \sum \alpha_{i}\left\|\left[h, f_{\varepsilon_{i}-1}\left(x_{i}\right)\right]\right\| \leqq 2^{-n}
$$

so that $\left\|\left[h-h_{n}, h_{k}\right]\right\| \leqq 2^{-n}$ for all $k \leqq n$, and the proof is complete. 
Remark. The preceding lemma shows that the infinitesimal generator for a group with spectrum condition is an inner limit derivation in the sense of Bratteli and Robinson.

We are indebted to H. Araki for pointing out the relevance of the next result for our problem, and for providing the following proof, which is included for completeness.

Lemma 5. Let $\left\{\alpha_{t}\right\}$ be a continuous one-parameter group of automorphisms of a $C^{*}$-algebra A, implemented by the unitary group $\{\exp (i t h)\}$, where $h$ is a self-adjoint operator (possibly unbounded). If $a$ and $k$ are smooth elements in $A$ and $k=k^{*}$, then with $\left\{\beta_{t}\right\}$ as the automorphism group implemented by the unitary group $\{\exp (i t k)\}$ we have

$$
\left\|\alpha_{t}(a)-\beta_{t}(a)\right\| \leqq|t|\|[h-k, a]\|+t^{2}\|[h, k]\|\|a\| .
$$

Proof. Let $\delta$ be the unbounded derivation given by $\operatorname{ad}(h-k)$. Then $i \delta=$ $d /\left.d t\left(\alpha_{t}-\beta_{t}\right)\right|_{t=0}$. However, we also have

$$
d /\left.d t\left(\alpha_{t} \circ \beta_{-t}\right)\right|_{t=0}=\lim t^{-1}\left(\alpha_{t} \circ \beta_{-t}-l\right)=\lim t^{-1}\left(\alpha_{t}-\beta_{t}\right) \circ \beta_{-t}=i \delta \circ l=i \delta .
$$

Consequently,

$$
\begin{aligned}
\alpha_{t}-\beta_{t} & =\int_{0}^{t} d /\left.d r\left(\alpha_{r} \circ \beta_{t-r}\right)\right|_{r=s} d s \\
& =\int_{0}^{t} d /\left.d r\left(\alpha_{s+r} \circ \beta_{t-s-r}\right)\right|_{r=0} d s \\
& =\int_{0}^{t} \alpha_{s} \circ d /\left.d r\left(\alpha_{r} \circ \beta_{-r}\right)\right|_{r=0} \circ \beta_{t-s} d s \\
& =\int_{0}^{t} \alpha_{s} \circ i \delta \circ \beta_{t-s} d s=i \int_{0}^{t} \alpha_{t-s} \circ \delta \circ \beta_{s} d s .
\end{aligned}
$$

Inserting the unitary groups in this formula we get

$$
\begin{aligned}
\left\|\alpha_{t}(a)-\beta_{t}(a)\right\| & \leqq \int_{0}^{t}\left\|\delta \circ \beta_{s}(a)\right\| d s \\
& =\int_{0}^{t}\|\delta(\exp (i s k) a \exp (-i s k))\| d s \\
& \leqq \int_{0}^{t}(\| \delta(\exp (i s k)\|\| a\|+\| \delta(a)\|+\| a\|\| \delta(\exp (-i s k)) \|) d s \\
& =|t|\|[h-k, a]\|+2\|a\| \int_{0}^{t} \|[h-k, \exp (i s k] \| d s \\
& \leqq|t|\|[h-k, a]\|+2\|a\| \int_{0}^{t}|s|\|[h, k]\| d s \\
& =|t|\|[h-k, a]\|+t^{2}\|a\|\|[h, k]\| .
\end{aligned}
$$

Lemma 6. Let $\pi: B \rightarrow A$ be a surjective morphism between separable $C^{*}$-algebras $A$ and $B$. If $\left\{x_{n}\right\}$ is an increasing sequence in $A_{+}$such that $x_{n}>x$, where $x$ is a selfadjoint operator affiliated with $A^{\prime \prime}$, then there is an increasing sequence $\left\{y_{n}\right\}$ in $B_{+}$ such that $\pi\left(y_{n}\right)=x_{n}$ for each $n$, and $y_{n} \gamma y$ where $y$ is a self-adjoint operator affiliated with $B^{\prime \prime}$.

Proof. Extending $\pi$ to a normal morphism $\pi^{\prime \prime}$ of $B^{\prime \prime}$ onto $A^{\prime \prime}$ we have $\operatorname{ker} \pi^{\prime \prime}=B^{\prime \prime} p$, where $p$ is an open central projection in $B^{\prime \prime}$. Since $\operatorname{ker} \pi$ is separable there is an element $b$ in $(\operatorname{ker} \pi)_{+}$such that $p$ is the range projection of $b(b$ is a strictly positive element). Using spectral theory on $b$ we can then find an approximate unit $\left\{u_{n}\right\}$ for $\operatorname{ker} \pi$ such that $u_{n} u_{m}=u_{m}$ for all $n>m$. 

and

Suppose that we have already chosen $y_{m}$ in $B_{+}$for all $m \leqq n$ such that $\pi\left(y_{m}\right)=x_{m}$

$$
y_{m-1} \leqq y_{m} \leqq \sum_{k=1}^{m}\left(1-u_{k}\right)\left\|x_{k}\right\|
$$

for all $m \leqq n$. Since

$$
y_{n} \leqq \sum_{k=1}^{n+1}\left(1-u_{k}\right)\left\|x_{k}\right\|
$$

and moreover

$$
\pi\left(y_{n}\right)=x_{n} \leqq x_{n+1} \leqq \sum_{k=1}^{n+1}\left\|x_{k}\right\|=\pi\left(\sum_{k=1}^{n+1}\left(1-u_{k}\right)\left\|x_{k}\right\|\right)
$$

we conclude from [8, Prop. 5] that there is a $y_{n+1}$ in $B$ with $\pi\left(y_{n+1}\right)=x_{n+1}$ and

$$
y_{n} \leqq y_{n+1} \leqq \sum_{k=1}^{n+1}\left(1-u_{k}\right)\left\|x_{k}\right\| \text {. }
$$

We can thus by induction find an increasing sequence $\left\{y_{n}\right\}$ in $B_{+}$satisfying the conditions above.

From spectral theory we see that the operator

$$
z=\sum_{k=1}^{\infty}\left(1-u_{k}\right)\left\|x_{k}\right\| p
$$

corresponds to an unbounded continuous function on $\operatorname{Sp}(b) \backslash\{0\}$, and consequently defines a self-adjoint operator affiliated with $B^{\prime \prime} p$ [note that for each $\lambda$ in $\operatorname{Sp}(b) \backslash\{0\}$ we have $u_{k}(\lambda)=1$ except for a finite number of $k$ 's]. Since $y_{n} p \leqq z$ for each $n$ we conclude that $y_{n} p \nearrow y^{\prime}$ for some self-adjoint operator $y^{\prime}$ affiliated with $B^{\prime \prime} p$. On the other hand $\pi^{\prime \prime}$ is a spatial isomorphism of $B^{\prime \prime}(1-p)$ onto $A^{\prime \prime}$ and therefore $y_{n}(1-p) \nearrow y^{\prime \prime}$ for some self-adjoint operator $y^{\prime \prime}$ affiliated with $B^{\prime \prime}(1-p)$. It follows that $y=y^{\prime}+y^{\prime \prime}$ is a self-adjoint operator affiliated with $B^{\prime \prime}$ such that $y_{n} \succ y$.

\section{Main Results}

Theorem 1.A continuous one-parameter group $\left\{\alpha_{t}\right\}$ of automorphisms of a separable $C^{*}$-algebra $A$ satisfies a universal spectrum condition if and only if there is an increasing sequence $\left\{h_{n}\right\}$ in $A_{+}$such that

(i) $\left\|\alpha_{t}(a)-\exp \left(i t h_{n}\right) a \exp \left(-i t h_{n}\right)\right\| \rightarrow 0$

uniformly on compact subsets of $\mathbb{R}$ for each a in $A$.

(ii) For each state $\omega$ of $A$ there is an $\varepsilon>0$ such that $\omega\left(\left(1+h_{n}\right)^{-1}\right) \geqq \varepsilon$ for all $n$.

Proof. If $\left\{\alpha_{t}\right\}$ satisfies a spectrum condition choose a dense sequence $\left\{a_{k}\right\}$ of smooth elements and a sequence $\left\{h_{n}\right\}$ satisfying Lemma 4 . Then by Lemma 5

$$
\left\|\alpha_{t}\left(a_{k}\right)-\exp \left(i t h_{n}\right) a_{k} \exp \left(-i t h_{n}\right)\right\| \leqq 2^{-n}\left(|t|+t^{2}\left\|a_{k}\right\|\right)
$$

for all $k \leqq n$. This implies condition (i) for each $a$ in $\left\{a_{k}\right\}$. However, $\left\{a_{k}\right\}$ is dense, and the difference between two automorphisms is bounded (by 2 ) so (i) holds for every $a$ in $A$.

Condition (ii) is equivalent with the demand that the strong limit $x$ in $A^{\prime \prime}$ of the decreasing sequence $\left\{\left(1+h_{n}\right)^{-1}\right\}$ satisfies $\omega(x)>0$ for every state $\omega$ of $A$. 
Thus condition (ii) merely says that $x$ must not have zero as an eigenvalue in the universal representation of $A$. Since in our case $h_{n}$ Th we have $x=(1+h)^{-1}$ by Lemma $A$ and (ii) is satisfied.

Conversely, if both (i) and (ii) are satisfied define $x$ as the limit of $\left\{\left(1+h_{n}\right)^{-1}\right\}$. Then by (ii) $h=x^{-1}-1$ is a self-adjoint positive operator affiliated with $A^{\prime \prime}$ and $h_{n}$ Th. It follows from Lemma A that $\exp \left(i t h_{n}\right) \rightarrow \exp (i t h)$ strongly, and since the norm is strongly lower semi-continuous condition (i) implies that $\left\{\alpha_{t}\right\}$ is implemented by $\{\exp (i t h)\}$ and therefore satisfies a spectrum condition.

Remark $A$. Condition (ii) in Theorem 1 may be replaced by the formally weaker assumption that for every pure state of $A$ there is an $\varepsilon>0$ such that $\omega\left(\left(1+h_{n}\right)^{-1}\right) \geqq \varepsilon$ for all $n$. Indeed, if $x$ is the strong limit in $A^{\prime \prime}$ of the decreasing sequence $\left\{\left(1+h_{n}\right)^{-1}\right\}$ then $x$ is of first Baire class regarded as an affine function on the state space of $A$, and by assumption $\omega(x)>0$ for every pure state. Since each state can be represented as a barycenter of pure states and since $x$, being of first Baire class, satisfies the barycenter formula we conclude that $\omega(x)>0$ for every state $\omega$ of $A$.

Remark $B$. The evaluations of the norms of $\left[h-h_{n}, a_{k}\right]$ and $\left[h-h_{n}, h_{k}\right]$ given in Lemma 4 are more precise than needed for the proof of Theorem 1 (but will be necessary for the proof of Theorem 2). In fact, since pointwise norm convergence of ad $h_{n}$ to $\delta$ on the core of smooth elements entails resolvent convergence, we could conclude group convergence directly from the Trotter-Kato theorem.

Remark $C$. Condition (i) in the preceding theorem says that $\left\{\alpha_{t}\right\}$ is an "approximately inner" group in the sense of Powers and Sakai. They show in [12, Theorem 2.3] that a $C^{*}$-algebra with unit and with an approximately inner oneparameter group of automorphisms satisfies a spectrum condition in at least one covariant representation. It may be of interest to note that their result can be obtained by straightforward applications of spectral subspace techniques: As in $[6,3.5 .2]$ let $p_{t}$ denote the left annihilating projection in $A^{\prime \prime}$ of the spectral subspace $R^{\alpha}(t, \infty)$. Thus $\left(1-p_{t}\right) A^{\prime \prime}$ is the smallest weak* closed right ideal containing $R^{\alpha}(t, \infty)$. A state $\omega$ which annihilates $R^{\alpha}(0, \infty) A$ is called a ground state in [12].

We see that $\omega$ is a ground state if and only if $\omega\left(p_{0}\right)=1$. Clearly then the support of the representation $\pi_{\omega}$ in $A^{\prime \prime}$ is majorized by $\vee p_{t}$, whence $\pi_{\omega}$ is covariant with spectrum condition by $[6,3.5 .3]$. A ground state is necessarily $\alpha$-invariant since $\omega\left(R^{\alpha}(0, \infty)\right)=0$ implies $\omega \in M^{\alpha^{\prime}}(-\infty, 0]$ (notations as in $\left.[6,3.4]\right)$. But then since $\omega=\omega^{*}$

$$
\omega \in M^{\alpha^{\prime}}(-\infty, 0] \cap M^{\alpha^{\prime}}[0, \infty)=M^{\alpha^{\prime}}\{0\},
$$

and $M^{\alpha^{\prime}}\{0\}$ is the fixed points of $\left\{\alpha_{t}^{\prime}\right\}$.

If $\left\{\alpha_{t}\right\}$ is approximately inner then $A$ has a ground state. Otherwise $p_{0}=0$ which means that the right ideal generated by $R^{\alpha}(0, \infty) A$ contains invertible elements. But then there is an inner group $\left\{\beta_{t}\right\}$ approximating $\left\{\alpha_{t}\right\}$ such that the right ideal generated by $R^{\beta}(0, \infty) A$ contains invertible elements, i. e. $p_{0}^{\beta}=0$. However, ground states always exist for inner groups.

Theorem 2. Let $\pi: B \rightarrow A$ be a surjective morphism between separable $C^{*}$-algebras $A$ and $B$. If $\left\{\alpha_{t}\right\}$ is a continuous one-parameter group of automorphisms of $A$ with 
spectrum condition then there is a continuous one-parameter group $\left\{\bar{\alpha}_{i}\right\}$ of automorphisms of $B$ with spectrum condition such that $\pi \circ \alpha_{t}=\bar{\alpha}_{t} \circ \pi$ for all $t$ in $\mathbb{R}$.

Proof. Since $\pi$ is an open map there is a dense sequence $\left\{b_{k}\right\}$ in $B$ such that each element $a_{k}=\pi\left(b_{k}\right)$ is smooth in $A$. Then with $h$ as the minimal positive generator for $\left\{\alpha_{t}\right\}$ we choose an increasing sequence $\left\{h_{n}\right\}$ in $A_{+}$satisfying Lemma 4 . By Lemma 6 there is then an increasing sequence $\left\{y_{n}\right\}$ in $B_{+}$with $\pi\left(y_{n}\right)=h_{n}$ for each $n$ and $y_{n} \succ y$ for some self-adjoint positive operator $y$ affiliated with $B^{\prime \prime}$. We may assume that $y_{1}=0$ and $h_{1}=0$.

Now let $\left\{u_{\lambda}\right\}$ be an approximate unit for $\operatorname{ker} \pi$ which at the same time is a convex set (The convex hull of any approximate unit is again an approximate unit with the ordering that one convex combination precedes another, if each of its components precedes any component of the other.). Define

$$
z_{n}=\left(y_{n+1}-y_{n}\right)^{\frac{1}{2}}\left(1-u_{\lambda}\right)\left(y_{n+1}-y_{n}\right)^{\frac{1}{2}} .
$$

We claim that it is possible for each $n$ to choose $u_{\lambda}$ such that with $x_{m}=\sum_{n=1}^{m} z_{n}$ (whence $\left.\pi\left(x_{m}\right)=h_{m+1}\right)$ we have

(*) $\left\|\left[z_{n}, b_{k}\right]\right\| \leqq 2^{1-n} \quad$ and $\quad\left\|\left[z_{n}, x_{k}\right]\right\| \leqq 2^{1-n}$

for all $k \leqq n$. Suppose that this has been established for all $z_{m}$ with $m<n$. Then for fixed $n$ and $k$ consider the net with elements

$$
\left[z_{n}, b_{k}\right]-\left(1-u_{\lambda}\right)\left[y_{n+1}-y_{n}, b_{k}\right] .
$$

Since $\left\{u_{\lambda}\right\}$ increases to a central projection in $B^{\prime \prime}$ (viz. the support of ker $\pi^{\prime \prime}$, cf. the proof of Lemma 7) this net is $\sigma\left(B, B^{\prime}\right)$ convergent to zero. Since the net is also convex it contains zero as a limit point in norm (reasoning as in the proof of Lemma 2). On the other hand

$$
\begin{aligned}
\left\|\left(1-u_{\lambda}\right)\left[y_{n+1}-y_{n}, b_{k}\right]\right\| & \left.\rightarrow \| \pi\left(y_{n+1}-y_{n}, b_{k}\right]\right) \| \\
& =\left\|\left[h_{n+1}-h_{n}, a_{k}\right]\right\|<2^{1-n},
\end{aligned}
$$

for all $k \leqq n$. We may therefore choose $u_{\lambda}$ such that $\left\|\left[z_{n}, b_{k}\right]\right\|<2^{1-n}$ for all $k \leqq n$. With exactly the same arguments we obtain

$$
\begin{gathered}
\left\|\left[z_{n}, x_{k}\right]\right\|<2^{-1-n}+\lim \left\|\left(1-u_{\lambda}\right)\left[y_{n+1}-y_{n}, x_{k}\right]\right\|=2^{-1-n}+\left\|\pi\left(\left[y_{n+1}-y_{n}, x_{k}\right]\right)\right\| \\
=2^{-1-n}+\left\|\left[h_{n+1}-h_{n}, h_{k+1}\right]\right\| \leqq 2^{1-n}
\end{gathered}
$$

for all $k \leqq n$, which proves the claim.

The sequence $\left\{x_{n}\right\}$ is increasing and since $x_{n} \leqq y_{n}$ for each $n$ we conclude that $x_{n}>x$ for some self-adjoint positive operator $x$ affiliated with $B^{\prime \prime}$. Using Lemma 5 and the inequalities $(*)$ we obtain for all $k \leqq n$ and all $m$

$$
\begin{aligned}
& \| \exp \left(\text { it } x_{n+m}\right) b_{k} \exp \left(-i t x_{n+m}\right)-\exp \left(\text { it } x_{n}\right) b_{k} \exp \left(-i t x_{n}\right) \| \\
& \quad \leqq|t|\left\|\left[x_{n+m}-x_{n}, b_{k}\right]\right\|+t^{2}\left\|b_{k}\right\|\left\|\left[x_{n+m}, x_{n}\right]\right\| \\
& \quad \leqq|t| \sum_{p=n}^{m} 2^{1-p}+t^{2}\left\|b_{k}\right\| \sum_{p=n}^{m} 2^{1-p} \\
& \leqq 2^{2-n}\left(|t|+t^{2}\left\|b_{k}\right\|\right)
\end{aligned}
$$


Since $\exp \left(i t x_{n+m}\right)$ converges strongly to $\exp (i t x)$ when $m \rightarrow \infty$, and the norm is strongly lower semi-continuous we conclude that

$$
\begin{gathered}
\left\|\exp (i t x) b_{k} \exp (-i t x)-\exp \left(i t x_{n}\right) b_{k} \exp \left(-i t x_{n}\right)\right\| \\
\leqq 2^{2-n}\left(|t|+t^{2}\left\|b_{k}\right\|\right)
\end{gathered}
$$

for all $k \leqq n$. It follows immediately from this that $x$ is the infinitesimal generator for a continuous one-parameter group of automorphisms $\left\{\bar{\alpha}_{t}\right\}$ of $B$ with spectrum condition (since $x \geqq 0$ ) such that $\pi\left(\bar{\alpha}_{t}(b)\right)=\alpha_{t}(\pi(b))$ for each $b$ in $B$ and $t$ in $\mathbb{R}$.

\section{References}

1. Araki,H., Woods, E.J.: Topologies induced by representations of the canonical commutation relations. Rep. Math. Phys. 4, $227-254$ (1973)

2. Arveson, W. B.: On groups of automorphisms of operator algebras. J. Funct. Anal. 15, 217-243 (1974)

3. Borchers, H.J.: On groups of automorphisms with semibounded spectrum. In: Systèmes à une numbre infini de degrés de liberté. Colloques Gif-sur-Yvette, Mai 1969 (C.N.R.S.)

4. Borchers, H. J.: Über Ableitungen von $C^{*}$-Algebren. Nachr. d. Göttinger Akademie. 1973, Nr. 2

5. Dixmier, J.: Les $C^{*}$-algèbres et leurs représentations. Paris: Gauthier-Villars 1964

6. Olesen, D.: On spectral subspaces and their applications to automorphism groups. To appear in: $C^{*}$-algebras and their applications in theoretical physics. Conference Rome, March 1975

7. Olesen, D., Pedersen, G.K.: Derivations of $C^{*}$-algebras have semi-continuous generators. Pac. J. Math. 53, 563-573 (1974)

8. Pedersen, G. K.: A decomposition theorem for $C^{*}$-algebras. Math. Scand. 22, 266-268 (1968)

9. Pedersen, G. K.: Lifting derivations from quotients of separable $C^{*}$-algebras. Proc. Nat. Acad. Sci. USA 73, $1414-1415$ (1976)

10. Pedersen, G.K.: Lifting groups of automorphisms. To appear in: $C^{*}$-algebras and their applications in theoretical physics. Conference Rome, March 1975

11. Pedersen, G.K., Takesaki,M.: The Radon-Nikodym theorem for von Neuman algebras. Acta Math. 130, 53-87 (1973)

12. Powers, R.T., Sakai, S.: Existence of ground states and KMS states for approximately inner dynamics. Commun. math. Phys. 39, 273-288 (1975)

Communicated by H. Araki

Received August 18, 1975; in revised form April 28, 1976 
